\title{
Statistical Analysis of CQI Reporting and MIMO Utilization for Downlink Scheduling in Live LTE Mobile Network
}

\author{
Igor A. Tomić, Đorđe Lukić, Milutin Davidović, Dejan D. Drajić, and Predrag Ivaniš
}

\begin{abstract}
This paper considers a Multiple Input Multiple Output (MIMO) downlink scheduling procedure in Long Term Evolution (LTE) cellular networks. In particular, the relationship between Channel Quality Indicator (CQI) and MIMO utilization in LTE Frequency Division Duplex (FDD) systems was examined using the statistical analysis. Data was collected from a mature mobile network deployed in typical frequency bands for LTE FDD used in Europe - Band 3, Band 7 and Band 20. Obtained numerical results showed a significant correlation between reported CQI value and MIMO utilization.
\end{abstract}

Keywords - CQI (Channel Quality Indicator), Downlink Scheduling, LTE (Long Term Evolution), MIMO (Multiple Input Multiple Output).

\section{INTRODUCTION}

$\mathrm{D}$ OWNLINK transmission in Long Term Evolution (LTE) cellular networks is based on Orthogonal Frequency Division Multiple Access (OFDMA) technique which enables flexible radio resource allocation. These physical radio resources are represented as a time-frequency grid consisting of multiple Resource Blocks (RB). RB is the smallest radio resource unit that can be allocated to a User Equipment (UE) for data transmission [1], [2]. LTE cellular networks using OFDMA, do not suffer any intracell interference like systems of earlier generations where multiple access is based on code multiplex, like Wideband Code Division Multiple Access (WCDMA). Furthermore, LTE provides much more spectrum flexibility, as it is easier to combine layers in different frequency bands deployed

Paper received May 02, 2020; accepted June 12, 2020. Date of publication July 31,2020 . The associate editor coordinating the review of this manuscript and approving it for publication was Prof. Zorica Nikolić.

This paper is revised and expanded version of the paper presented at the 27th Telecommunications Forum TELFOR 2019 [10].

Igor A. Tomić, Aspire Technology Unlimited - Belgrade Branch Office, Vladimira Popovića 6, Unit B10, 11070 Belgrade, Serbia (e-mail: igor.tomic@aspiretechnology.com).

Đorđe Lukić, Aspire Technology Unlimited - Belgrade Branch Office, Vladimira Popovića 6, Unit B10, 11070 Belgrade, Serbia (e mail: djordje.lukic@aspiretechnology.com).

Milutin Davidović, Ericsson Spain, Calle Retama 1, 28045 Madrid, Spain (e mail: milutin.davidovic@ericsson.com).

Dejan Drajić, School of Electrical Engineering, University of Belgrade, Bulevar Kralja Aleksandra 73, 11000 Belgrade, Serbia (e-mail: ddrajic@etf.bg.ac.rs).

Predrag Ivaniš, School of Electrical Engineering, University of Belgrade, Bulevar Kralja Aleksandra 73, 11000 Belgrade, Serbia (e-mail: predrag.ivanis@etf.bg.ac.rs). with different bandwidths. Finally, LTE improves spectrum efficiency compared to WCDMA systems, mainly by Multiple Input Multiple Output (MIMO) technology introduction, that also achieves higher peak data rates with multiple antennas deployed on both transmitter and receiver side.

In the last few years mobile operators have faced data tsunami, where the amount of data transmitted over mobile networks was growing exponentially. Situation is different from market to market, but it is quite often that payload is doubling year over year. Worth mentioning is that there are new use cases that mobile operators are introducing, mainly in the area of Internet of Things (IoT), that are increasing network load further [3]. In such circumstances, LTE cell capacity and spectrum efficiency are important more than ever [4], [5].

MIMO utilization is an important driver for spectrum efficiency, and the main goal of this paper is to investigate its correlation with interference in the system, measured by Channel Quality Indicator (CQI). The paper is organized as follows. Section II introduces the theoretical background for Channel State Information (CSI) reporting and operating MIMO system model. Challenges of real network deployment, that are one of the main motivations for this research, have been discussed in Section III. The statistical approach used for examination of this dependency and obtained results in terms of statistical dependency between reported CQI value and MIMO utilization in different radio conditions are elaborated in Section IV. Finally, conclusions and future work are given in Section V.

\section{THEORETICAL BACKGROUND}

MIMO provides three operational modes: transmit diversity, beamforming and spatial multiplexing.

In transmit diversity mode, typically Space-Time Block Code (STBC) such as Alamouti is used, or open-loop precoder cycling. Channel knowledge is not required. This can be useful for high mobility scenarios when rapid channel changes cannot be tracked. Transmit diversity does not increase peak data rate but reduces the probability of fading dip and optimizes Bit Error Rate (BER) vs Signal to Noise Ratio (SNR) curve, decreasing BER and improving system robustness.

Beamforming is used to improve directivity towards user and SNR, where $N$ antennas theoretically may increase SNR with a factor up to $N$. Increased SNR improves data rates and user experience but only under a logarithmic 
function. Beamforming benefits for user experience in high SNR conditions are not major, but on cell edge with a low SNR may be significant. As beamforming requires deep channel state knowledge to determine precoder weights, it may be applicable only in low mobility scenarios, when required up-to date channel information is available.

Spatial multiplexing enables transmission of several independent data streams over the same time-frequency resources. As overall available transmission power is shared between streams, SNR will be decreased. However, in good SNR conditions, significant benefits may be achieved, as MIMO spatial multiplexing helps to achieve system capacity beyond the Shannon limit.

From the information theory, in case of $N \mathrm{x} N \mathrm{MIMO}$ system, spatial multiplexing can replace one stream that has $N$ times higher SNR with multiple parallel streams that have $N$ times lower SNR. In such a case the total channel capacity will be improved since it is proportional to $N \log (1+S N R)$ as can be seen in Fig. 1 [6].

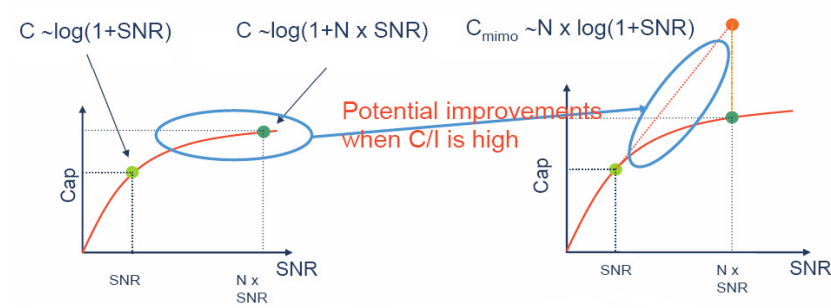

Fig. 1. MIMO Spatial multiplexing improves channel capacity and Shannon limit.

Typically, in advanced LTE systems, especially with massive MIMO and a high number of antenna elements, some of the three described MIMO modes are combined. An example of a frequently used combination is beamforming and spatial multiplexing, where some degrees of freedom are spent to increase directivity (which improves SNR) and remaining ones are spent to increase the capacity (which requires a larger SNR). However, it should be noticed that in LTE $2 \times 2$ MIMO systems, widely deployed these days in operating commercial LTE networks around the globe, the most significant benefit for network performance and capacity is coming from $2 \times 2$ spatial multiplexing.

Let us assume that in a LTE $2 \times 2$ MIMO system $N_{R I 1}$ and $N_{R I 2}$ denote the number of transmissions whenever UE is scheduled with a new downlink data and used rank is 1 and 2, respectively. MIMO downlink utilization of the analyzed system is defined as:

$$
M I M O_{D L \text { Utilization }}=N_{R I 2} /\left(N_{R I 1}+N_{R I 2}\right)
$$

and it represents the percentage of transmissions in which the number of parallel transmission layers is equal to two, which is a maximum in this case. Analyzing the impact of MIMO utilization on system performance, clear correlation with spectrum efficiency may be noticed. Spectral efficiency is expressed in $\mathrm{bps} / \mathrm{Hz}$ and measured as the number of bits transmitted at Packet Data Convergence Protocol (PDCP) layer per second in a cell over an average number of RBs used. A typical example of measurements from commercial LTE $2 \times 2$ network are presented in Fig. 2, where almost linear correlation is evident.

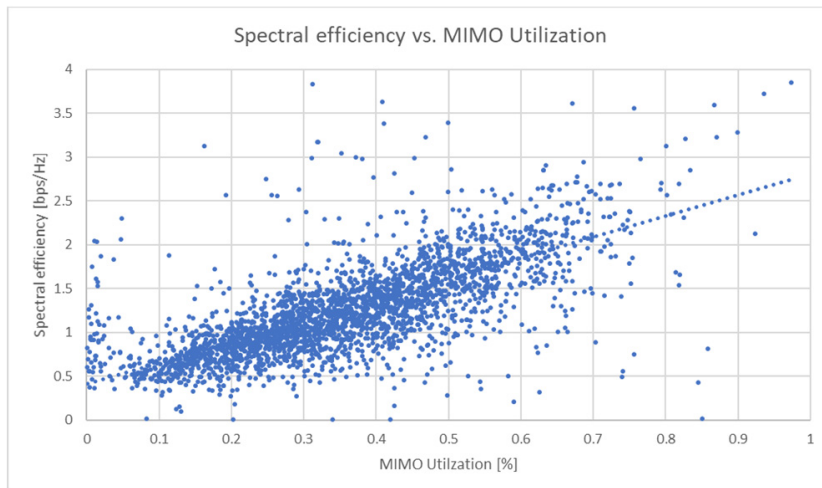

Fig. 2. Spectral Efficiency vs MIMO Utilization, example from commercial LTE 2x2 network.

As MIMO utilization is obviously one of the most relevant drivers for overall LTE performance, it is important to understand its evolution over time with growing network load. In this paper the CQI reporting for downlink scheduling procedure in practical LTE systems and its impact on MIMO communication will be analyzed.

The critical part of the whole process is happening in a downlink scheduler located in eNB, which needs to know radio conditions for each UE. To enable the process of estimation on the state of MIMO channel and selection of optimal transmission scheme, 3GPP standard requests each UE to perform CSI reporting that carries necessary information on a radio signal [7].

CSI has three major components: Channel Quality Indicator (CQI), Precoding Matrix Index (PMI) and Rank Indicator (RI). CSI reporting may be periodic and aperiodic. Periodic reporting is carried either by Physical Uplink Control Channel (PUCCH) or Physical Uplink Shared Channel (PUSCH) when user data on Dedicated Control Channel (DCCH) and Dedicated Traffic Channel (DTCH) needs to be transmitted in the same time with L1/L2 control signaling. Periodicity is configured by a higher Radio Resource Control (RRC) layer. Aperiodic reporting is used mostly for Random Access Channel (RACH) procedure, where handovers or losses of synchronization are common reasons. Aperiodic reporting also happens when eNodeB schedules specific Physical Downlink Control Channel (PDCCH) Downlink Control Indicator (DCI) format 0 together with CQI request field set to "1", demanding uplink grant for UE data together with an aperiodic CQI report.

CQI implicitly indicates a suitable link adaptation and downlink transmission data rate, i.e. the highest Modulation and Coding Scheme (MCS) value at which the UE will be able to properly demodulate and decode the downlink data at target Block Error Rate (BLER) of 10\%. CQI accepts discrete values between 0 and 15 , where index 15 indicates the best channel quality and index 0 indicates the poorest channel quality.

What is important to indicate is that CQI estimation process should always take into account UE capability (i.e. number of antennas, type and capability of receiver used for detection, etc.) in order to facilitate eNode-B to make a proper selection on optimum MCS level for the transmission. This is important since, for the same SINR value, the MCS value to be fitted (supported) by a UE 
depends on these various UE capabilities.

An example of CQI tabular values for Modulation scheme up to 64QAM defined by 3GPP [7] is presented in Table 1. It can be noticed that with a higher CQI, higher order modulation is used, while coding rate increases for the same modulation with CQI increase. Last column in table presents calculated overall efficiency, taking into consideration the number of bits per symbol for different modulations (2 for QPSK, 4 for 16QAM, 6 for 64QAM and 8 for 256 QAM).

\begin{tabular}{c|c|c|c}
\multicolumn{4}{c}{ TABLE 1: 4-BIT CQI TABLE UP TO 64QAM MODULATION. } \\
\hline CQI index & modulation & $\begin{array}{c}\text { code rate } \\
\boldsymbol{x ~ 1 0 2 4}\end{array}$ & efficiency \\
\hline 0 & & Out of range & \\
1 & QPSK & 78 & 0.1523 \\
2 & QPSK & 120 & 0.2344 \\
3 & QPSK & 193 & 0.3770 \\
4 & QPSK & 308 & 0.6016 \\
5 & QPSK & 449 & 0.8770 \\
6 & QPSK & 602 & 1.1758 \\
7 & 16QAM & 378 & 1.4766 \\
8 & 16QAM & 490 & 1.9141 \\
9 & 16QAM & 616 & 2.4063 \\
10 & 64QAM & 466 & 2.7305 \\
11 & 64QAM & 567 & 3.3223 \\
12 & 64QAM & 666 & 3.9023 \\
13 & 64QAM & 772 & 4.5234 \\
14 & 64QAM & 873 & 5.1152 \\
15 & 64QAM & 948 & 5.5547 \\
\hline
\end{tabular}

A similar table is defined for modulation scheme up to 256QAM. It is interesting to notice that vendor proprietary software in a scheduler may decide to use table for mapping CQI up to 64QAM modulation even for UEs capable of 256QAM. Typically, it is applied when 256QAM capable $\mathrm{UE}$ is in bad radio conditions and low chance of using high order modulation, with the idea to achieve better CQI reporting precision.

UE reports the number of MIMO layers supported in downlink with RI. The maximum number of layers that can be created depends on UE capability in terms of the number of transmit and receive antennas and on radio channel characteristics and ability to achieve statistically independent paths in propagation. Consider an LTE MIMO downlink system with $N_{T}$ transmit antennas and $N_{R}$ receive antennas that supports a closed-loop spatial multiplexing transmission mode (TM). Let $h_{i, j}$ model the channel transfer function between transmit antenna $j$ and receive antenna $i$. The $i$-th received channel vector is $\mathbf{h}_{i}=$ $\left[\mathrm{h}_{i, 1} \mathrm{~h}_{i, 2} \ldots \mathrm{h}_{i, N_{T}}\right]$ hence MIMO channel $\mathbf{H}$ forms the $N_{R} \mathrm{x} N_{T}$ matrix:

$$
\mathbf{H}=\left[\begin{array}{llll}
\mathbf{h}_{1}^{\mathrm{H}} & \mathbf{h}_{2}^{\mathrm{H}} & \ldots & \mathbf{h}_{N_{R}}^{\mathrm{H}}
\end{array}\right]
$$

where $(.)^{\mathrm{H}}$ denotes the matrix Hermitian operation. The quasi-static and flat-fading channel is assumed so the matrix $\mathbf{H}$ can be taken as invariant during the round-trip time (RTT) period.

The transmit signal vector is pre-coded in a base station with the weight matrix called precoding matrix before it is transmitted to cancel the user interference and enhance the MIMO system performance. Collecting the basebandequivalent outputs, the $N_{R} \mathrm{x} 1$ received signal vector $\mathbf{y}$ on $\mathrm{UE}$ side is:

$$
y=H W x+n
$$

where $\mathbf{x}$ denotes the $N_{R} \mathrm{x} 1$ transmit vector, $\mathbf{W}$ is $N_{T} \times N_{R}$ linear precoding matrix applied in eNB and $\mathbf{n}$ denotes the $N_{R} \mathrm{x} 1$ noise vector.

In order to determine downlink throughput for UE, eNB needs to know how many transmission layers are supported and MIMO channel configuration before resource mapping. In this stage of LTE downlink scheduling procedure, the information obtained from PMI and RI reporting play the essential role. Channel matrix $\mathbf{H}$ can be decomposed into three components using the Singular Value Decomposition (SVD) technique:

$$
\mathbf{H}=\mathbf{U} \mathbf{\Sigma} \mathbf{V}^{\mathrm{H}}
$$

where $\mathbf{U}$ is $N_{R} \mathrm{x} N_{R}$ unitary matrix, $\boldsymbol{\Sigma}$ is $N_{R} \mathrm{x} N_{T}$ non-negative diagonal matrix and $\mathbf{V}$ is $N_{T} \times N_{T}$ unitary matrix. Assuming the optimal filter in receiver that matches transmitted signal and maximizes SNR, based on the channel estimation result in (4) UE calculates the received signal as follows:

$$
\mathbf{y}=\mathbf{U}^{\mathrm{H}} \mathbf{H V} \mathbf{x}
$$

From (5) it can be shown that channel matrix $\mathbf{H}$ is expressed as a simple diagonal matrix $\boldsymbol{\Sigma}$. This is a very important conclusion because it allows UE to easily get information about the number of supported MIMO layers. The number of non-zero diagonal elements of matrix $\boldsymbol{\Sigma}$ represents the RI value that UE reports to eNB.

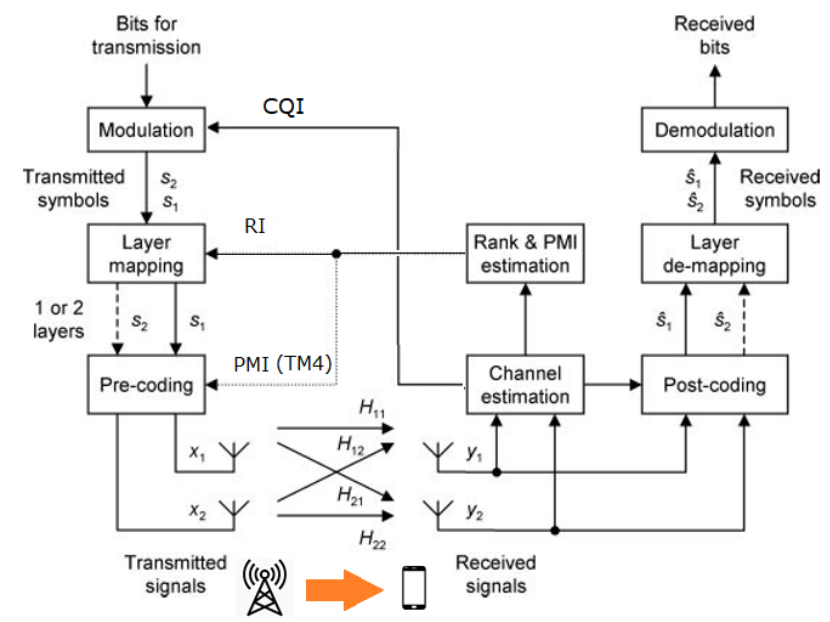

Fig. 3. LTE 2x2 MIMO - CSI reporting and DL scheduling process.

Upon receiving CSI, eNB determines required downlink throughput and allocates resources to UE. Modulation selection is done mainly based on reported CQI, layer mapping based on reported RI and precoding based on reported PMI. It is still possible to use $2 \times 2$ MIMO in open loop with Transmission Mode 3 (TM3), where PMI reporting is not required. In that case precoding is typically done in a way to use different available precoding matrix sequentially, to improve robustness of the system. Fig. 3 presents an overview of the whole process, [1]. 


\section{REAL NETWORK DEPLOYMENT}

In the real network deployment scenario, there will be inevitably some deviations from theoretical considerations. First it should be considered that LTE downlink scheduling procedure described in Section II assumes an invariant channel matrix $\mathbf{H}$, perfect CSI feedback and a precoding matrix $\mathbf{W}$ that fully cancels the interference generated from the other users in a cell. However, in practical LTE systems UE cannot perfectly estimate the channel conditions. Channel $\mathbf{H}$ is dynamically changing which results in an incomplete CSI report.

A few other relevant facts about practical realization should be noticed. Although UE through CSI report measurements and based on knowledge of radio propagation and channel conditions, proposes what considers as the optimal transmission, scheduler still doesn't need to obey the proposal. It may have a different decision, taking into consideration other relevant factors not known to UE, such as general conditions in the cell, traffic patterns, Quality of Service (QoS) among different UEs, etc. Also, realization of scheduler is not absolutely defined in 3GPP, leaving a certain amount of freedom for implementation to Radio Access Network (RAN) vendors and system designers.

The same principle applies on UE side, where chipset system designers again have a certain amount of freedom to optimize the process of CSI reporting to what they believe will provide a better performance. Important to understand is that criteria for what is considered a good performance and focus for optimization, will not necessarily be the same for network designers on one side, who are typically looking for improved spectrum efficiency, and UE designers on the other side, very focused, for instance, on battery consumption improvement. It should be noticed that different $U E$ in the same radio conditions may report different CQI or different RI.

When it comes to differences in CSI estimation process conducted in UEs, too optimistic or too pessimistic CQI reporting is compensated by eNB scheduler, that will adjust reported CQI through an additional loop where BLER value versus target $10 \%$ will be checked. However, when it comes to RI, scheduler is not able to apply a higher rank than UE requested. In previous work [8] it was observed that UE in poor radio conditions would tend to report Rank 1 .

This paper focuses on the investigation of dependences between CQI and MIMO utilization in a practical LTE downlink system and real environment in terms of network design, traffic patterns, UE capabilities distribution, etc. Observed LTE system allows up to two independent data streams between UE and eNB (LTE $2 \times 2$ MIMO) and operates in open loop regime with TM3.

\section{Statistical Analysis, Methodology And RESULTS}

Reported CQI and RI values are collected from the measurements performed in live mature LTE Frequency Division Duplex (FDD) networks deployed on typical frequency bands used in Europe: Band 20 (800 MHz), Band $3(1800 \mathrm{MHz})$ and Band 7 (2600 MHz).
Observed was a busy hour identified on a sector basis over an observation period ( 72 hours), where selected hour was the one with the highest amount of data traffic. Results for different LTE FDD bands are presented together in a graphical and tabular manner. MIMO downlink utilization is obtained using expression (1), where counters for the number of transmissions with different ranks have been collected for each cell in a mobile cellular system. Reported CQI value was averaged from CDF distribution of CQI reports collected for each cell during the period of observation.

Fig. 4 presents correlation between MIMO utilization and reported CQI for band 20 (low band $-800 \mathrm{MHz}$ ). At a first glance, a good level of correlation can be noticed between two indicators. It can be noticed that reported CQI range is mainly between values of 7 and 10 .

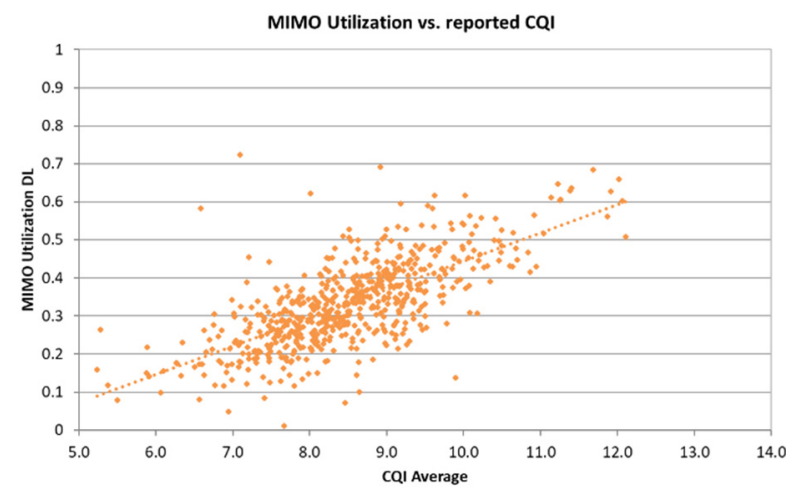

Fig. 4. MIMO Utilization vs. Reported CQI, LTE FDD Band 20.

The same dependence trend is kept in different bands, Fig 5 and Fig. 6 present results for band 3 (mid-band $1800 \mathrm{MHz}$ ) and band 7 (mid-band 2600MHz), respectively.

Observing correlations between MIMO Utilization and reported CQI for different frequency bands it can be noticed that while dependence is similar - linear like, reported CQI and corresponding MIMO Utilization is higher when moving to higher frequencies. For instance, reported CQI for Band 3 moved to range between values of 8 and 11 , while for band 7 it improved further and moved to range of values between 10 and 12. In general, reported CQI and corresponding MIMO Utilization are a result of system design and ability to control interference. One of the main reasons behind a better performance on higher frequencies is related to propagation of radio waves. As frequency increases, system is less sensitive to inter-cell interference, due to more challenging propagation and higher losses [9], attenuating undesirable signals from neighboring cells.

The next step is to quantify the level of correlation. The reported CQI and MIMO utilization are treated as two discrete random variables $X$ and $Y$ that form a random vector $(X, Y)$ with $N$ samples where $N$ is the size of a set of all possible results of these two random variables, or in a particular dataset the number of observed cells. The expected values and standard deviations for two variables are denoted by $\mu_{X}, \mu_{Y}$ and $\sigma_{X}, \sigma_{Y}$ respectively. The correlation coefficient $\rho_{X, Y}$ indicates the degree of linear dependency between $X$ and $Y$ : 


$$
\rho_{X, Y}=\frac{\mathrm{E}\left[\left(X-\mu_{X}\right)\left(Y-\mu_{Y}\right)\right]}{\sigma_{X} \sigma_{Y}}
$$

where $\mathrm{E}$ is the expected value operator. Method of moments provides the estimated value of correlation coefficient:

$$
\hat{\rho}=\frac{\sum_{k=1}^{N}\left(X_{k}-\hat{\mu}_{X}\right)\left(Y_{k}-\hat{\mu}_{Y}\right)}{\left(\sum_{k=1}^{N}\left(X_{k}-\hat{\mu}_{X}\right)^{2} \sum_{k=1}^{N}\left(Y_{k}-\hat{\mu}_{Y}\right)^{2}\right)^{1 / 2}}
$$

Correlation coefficient value is never bigger than 1. If random variables are independent their correlation coefficient is 0 , while as level of correlation increases, correlation coefficient value is always below 1 . The estimated correlation coefficient values for analyzed LTE FDD bands are presented in Table 2.

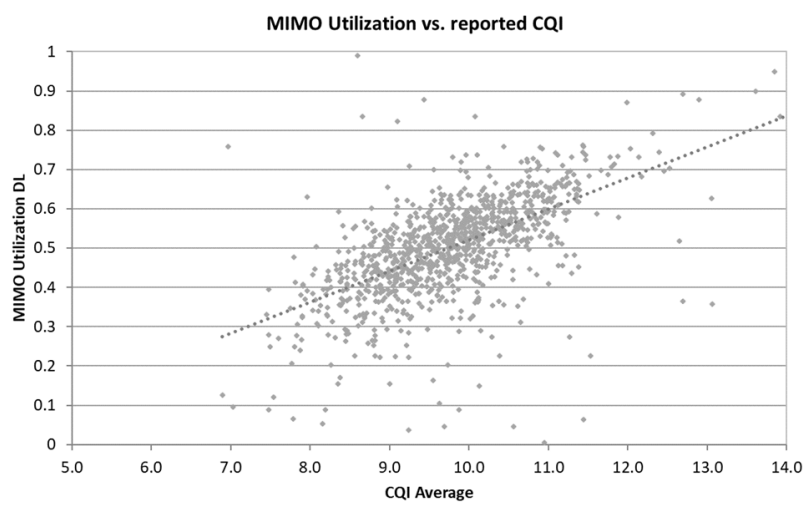

Fig. 5. MIMO Utilization vs Reported CQI, LTE FDD Band 3.

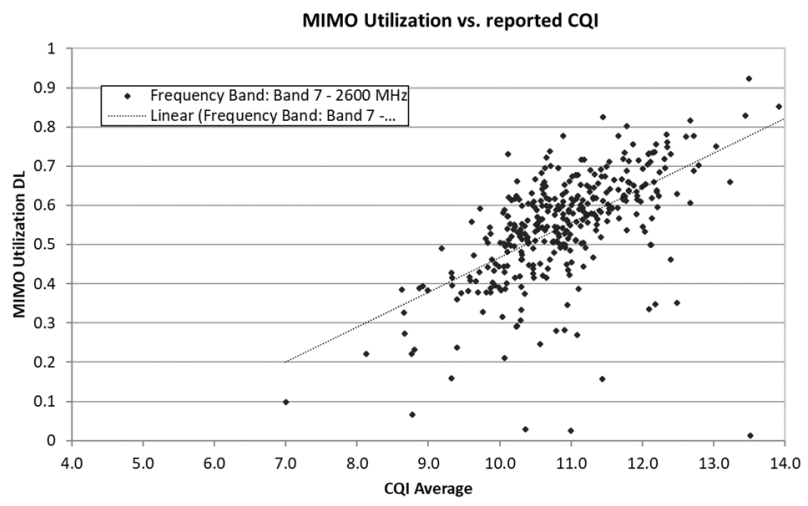

Fig. 6. MIMO Utilization vs Reported CQI, LTE FDD Band 7.

TABLE 2: ESTIMATED CORRELATION COEFFICIENT VALUES FOR ANALYZED LTE FDD BANDS.

\begin{tabular}{c|c|c}
\hline LTE FDD band & $\widehat{\boldsymbol{\rho}}$ & $\boldsymbol{N}$ \\
\hline Band $20(800 \mathrm{MHz})$ & 0.727 & 621 \\
Band $3(1800 \mathrm{MHz})$ & 0.670 & 980 \\
Band $7(2600 \mathrm{MHz})$ & 0.733 & 341 \\
\hline
\end{tabular}

Calculated correlation coefficient with values around 0.7 for all observed LTE FDD bands classifies MIMO utilization and reported CQI as two highly correlated variables. The number of samples is significant enough.

\section{CONCLUSION}

In this paper the dependence between reported CQI and MIMO utilization in live LTE $2 \times 2$ MIMO networks was studied.

The analysis was carried out for typical LTE FDD bands, and it points to a strong relation between the observed parameters. Results are showing that reported CQI value is highly correlated with MIMO utilization for different operating frequency bands, from a low band such as Band $20(800 \mathrm{MHz})$ to mid bands such as Band $3(1800 \mathrm{MHz})$ or Band 7 (2600 MHz).

Obtained results presented in this paper encourage further work in this area. The next steps would be to observe networks in a longer time period and analyze the evolution of MIMO utilization in a network with a growing load, where CQI degradation is expected. Networks with different levels of maturity and different traffic patterns, various deployed bands and configurations should be tackled to get the credible view of this research problem. The outcome of such an analysis could be very relevant for modelling of a mobile network performance and would be a solid base for understanding the network performance and its evolution over time, as traffic increases.

\section{REFERENCES}

[1] Cristopher Cox, An Introduction to LTE, $2^{\text {nd }}$ edition, John Wiley \& The Sons Ltd, Chichester 2014.

[2] N. Gospić, I. Tomić, D. Popović and D. Bogojević, "Razvoj mobilnih telekomunikacija, od GSM do LTE," Univerzitet u Beogradu, Saobraćajni fakultet, Beograd 2010.

[3] M. Davidović, I. Tomić, D. Drajić, and Z. Čiča, "On the Impact of NB-IoT on LTE MBB Downlink Performance," TELFOR Journal, Vol.11, No.1, pp. 20-24, Belgrade 2019.

[4] M.Davidović, S.Bjekovic and I.Tomic, "On the impact of network load on LTE network downlink performance", $59^{\text {th }}$ International Conference IcETRAN 2015, Srebrno Jezero 2015.

[5] I.A.Tomić, M.S.Davidović and S.M.Bjeković, "On the downlink capacity of LTE cell," $23^{\text {rd }}$ Telecommunications Forum TELFOR 2015, pp. 181-185, Belgrade 2015.

[6] I. Tomić, I. Simić: "3GPP LTE Long Term Evolution - 4G system," YU INFO 2009, Kopaonik 2009

[7] "Evolved Universal Terrestrial Radio Access (E-UTRA); Physical Layer Procedures," 3GPP TS 36.213 15.7.0, September 2019.

[8] A.Elnashar, M. A. El-Saidn, "Looking at LTE in Practice: A Performance Analysis of the LTE System Based on Field Test Results," IEEE Vehicular Technology Magazine, Vol. 8, pp. 81-92, September 2013.

[9] Radio Wave Propagation Guidelines, Ericsson Internal, 2016.

[10] I. A. Tomić, Đ. Lukić, M. Davidović, D. D. Drajić and P. Ivaniš, "Statistical Analysis of CQI Reporting and MIMO Utilization for Downlink Scheduling in Live LTE Mobile Network," 2019 27th Telecommunications Forum (TELFOR), Belgrade, Serbia, 2019, pp. 1-4, November 2019 